Discussion Paper Series A No.680

\title{
An Equitable Nash Solution to Nonconvex Bargaining Problems
}

Yongsheng $\mathrm{Xu}$

(Department of Economics, Andrew Young School of Policy Studies, Georgia State University)

and

Naoki Yoshihara

(Department of Economics, University of Massachusetts Amherst, Institute of Economic Research, Hitotsubashi University and School of Management, Kochi University of Technology)

October 2018

Institute of Economic Research

Hitotsubashi University

Kunitachi, Tokyo, 186-8603 Japan 


\title{
An equitable Nash solution to nonconvex bargaining problems*
}

\author{
Yongsheng $\mathrm{Xu}$ \\ Department of Economics \\ Andrew Young School of Policy Studies \\ Georgia State University, Atlanta, GA 30302 \\ Naoki Yoshihara \\ Department of Economics, University of Massachusetts Amherst, Amherst, \\ MA 01002, USA; \\ The Institute of Economic Research, Hitotsubashi University, Kunitachi, \\ Tokyo 186-0004 Japan; and \\ School of Management, Kochi University of Technology, Kochi 782-8502, \\ Japan.
}

This Version: July 2018

* An earlier version of the paper was presented at the SEA meetings in Atlanta, Georgia, November 2010 and at the CEPET meeting in Udine, Italy, June 2011. We are grateful to M.A. Ballester, Youngsub Chun, Marco Mariotti, Hans Peters, Koichi Tadenuma, and William Thomson for helpful and encouraging comments. We are also grateful to an Associate Editor and a referee for very helpful comments on an earlier version of the paper. 


\begin{abstract}
This paper studies the Nash solution to non-convex bargaining problems. Given the multiplicity of the Nash solution in this context, we refine the Nash solution by incorporating an equity consideration. The proposed refinement is defined as the composition of the Nash solution and a variant of the Kalai-Smorodinsky solution. We then present an axiomatic characterization of the new solution.
\end{abstract}

J.E.L. Classification Numbers: C71, C78, D6, D7

Keywords: non-convex bargaining problem, Nash solution, equitable Nash solution, equity principle, binary weak axiom of revealed preference 


\section{Introduction}

Non-convex bargaining problems can arise in many economic contexts of resource allocations where, for example, due to economies of scale in the production technology, the underlying set of feasible allocations is itself not convex, and/or randomization is unavailable due to the lack of correlating strategies among players. In this paper, we study solutions to such nonconvex bargaining problems. We are particularly interested in a solution that incorporates both equity and efficiency considerations.

The classical Nash solution (Nash (1950)) to bargaining problems is originally proposed for convex bargaining problems, though it has been extended to non-convex problems. For instance, Kaneko (1980) proposes an extension of the classical Nash solution to non-convex problems as the set of all Nashproduct maximizers from each bargaining problem, whereas Herrero (1989) proposes a solution that constitutes a superset of the solution proposed by Kaneko on each non-convex problem, and Conley and Wilkie (1996) propose a solution which is single-valued, continuous, but only weakly efficient in that domain. Finally, Zhou (1997) also proposes an extension of the classical Nash solution which is a single-valued, continuous, efficient, but is not anonymous.

Given these proposals, there are recent axiomatic studies on the Kaneko type of the Nash solution to nonconvex problems (Marriotti (1998, 1999, 2000), Xu and Yoshihara (2006), and Peters andVermeulen (2012)), and on the Kalai-Smorodinsky (1975) (KS) solution and the egalitarian solution (Conley and Wilkie (1991), and Xu and Yoshihara (2006)). These works suggest that in nonconvex problems, the Nash solution has a greater efficiency consideration than the KS and the egalitarian solutions while each of the KS and the egalitarian solutions has a greater equity consideration than the Nash solution. Among these three, since the KS and the egalitarian solutions are single-valued, it is impossible to consider a refinement of each of them to improve their performance in terms of efficiency. In contrast, the Nash solution a là Kaneko (1980) is multi-valued since, as Mariotti (1998, 1999) shows, there is no single-valued solution satisfying the Nash axioms in nonconvex problems. Due to this multi-valuedness of the Nash solution, it would be desirable to consider a refinement of this solution to exclude some options recommended by it, if in particular such options are deemed to be undesirable from the viewpoint of distributive fairness.

We propose and study a refinement of the Nash solution to nonconvex bargaining problems by introducing an equity axiom, called Equity Principle 
(EP) (see Section 5 for a formal definition). The underlying equity idea of this axiom is originated from the Hammond equity (Hammond (1976)) principle, which is taken as a natural and standard equity condition in many contexts of collective choice problems. Thus, the motivation behind our proposal is to make the proposed refinement select among the Nash maximizers the ones which have the property of the Hammond equity. The refinement, namely, the equitable Nash solution, is derived from (EP) and other properties motivated by the Nash type axioms, and is a composition of the Nash solution and a variant of the KS solution, in that it selects the maximizers of the Nash product at the first stage, and then select, among such maximizers, the outcomes in which the ratios of all players' utility gains with respect to their maximum attainable utilities are equalized. ${ }^{1}$ Given the nature of the Nash solution to non-convex bargaining problems, it is reasonable for a refinement of the Nash solution to adopt an equity concern as embedded in the KS solution, since the KS solution has the same informational requirement on individual utilities as the Nash solution does.

The remainder of the paper is organized as follows. In Section 2, we present notations and definitions. Section 3 introduces a set of our core axioms and studies their implications on nonconvex problems. Section 4 introduces the new solution, the equitable Nash solution. Section 5 is devoted to the study of this solution, and we conclude in Section 6 .

\section{Notation and definitions}

Let $N=\{1,2, \ldots, n\}$ be the set of players with $n \geq 2$. Let $\mathbf{R}_{+}$be the set of all non-negative real numbers and $\mathbf{R}_{++}$be the set of all positive numbers. Let $\mathbf{R}_{+}^{n}$ (resp. $\mathbf{R}_{++}^{n}$ ) be the $n$-fold Cartesian product of $\mathbf{R}_{+}$(resp. $\mathbf{R}_{++}$). For any $x, y \in \mathbf{R}_{+}^{n}$, we write $x \geq y$ to mean $\left[x_{i} \geq y_{i}\right.$ for all $\left.i \in N\right], x>y$ to mean $\left[x_{i} \geq y_{i}\right.$ for all $i \in N$ and $\left.x \neq y\right]$, and $x \gg y$ to mean $\left[x_{i}>y_{i}\right.$ for all $i \in N]$. For any $x \in \mathbf{R}_{+}^{n}$ and any non-negative number $\alpha$, we write $z=\left(\alpha ; \mathbf{x}_{-i}\right) \in \mathbf{R}_{+}^{n}$ to mean that $z_{i}=\alpha$ and $z_{j}=x_{j}$ for all $j \in N \backslash\{i\}$. For any subset $A \subseteq \mathbf{R}_{+}^{n}, A$ is said to be (i) non-trivial if there exists $a \in A$ such that $a \gg 0$, and (ii) comprehensive if for all $x, y \in \mathbf{R}_{+}^{n},[x>y$ and $x \in A]$

\footnotetext{
${ }^{1}$ Note that Conley and Wilkie (1996) propose an "equitable" Nash extension solution to nonconvex problems, which is taken as a hybrid of the Nash and the KS solutions. However, their solution is very different from ours proposed in this paper as their solution does not satisfy the efficiency criterion and is not a refinement of the Nash solution.
} 
implies $y \in A$. For all $A \subseteq \mathbf{R}_{+}^{n}$, define the comprehensive hull of $A$, to be denoted by compA, as follows:

$$
\operatorname{comp} A \equiv\left\{z \in \mathbf{R}_{+}^{n} \mid z \leq x \text { for some } x \in A\right\} .
$$

Let $\Sigma$ be the set of all non-trivial, compact and comprehensive subsets of $\mathbf{R}_{+}^{n}$. Elements in $\Sigma$ are interpreted as (normalized) bargaining problems. A problem $A \in \Sigma$ is called simple if there are $x, y \in A$ such that $A=$ comp $\{x, y\}$. A bargaining solution $F$ assigns a nonempty subset $F(A)$ of $A$ for every bargaining problem $A \in \Sigma$.

Let $\pi$ be a permutation of $N$. For all $x=\left(x_{i}\right)_{i \in N} \in \mathbf{R}_{+}^{n}$, and any permutation $\pi$, let $\pi(x)=\left(x_{\pi(i)}\right)_{i \in N}$. For all $A \in \Sigma$ and any permutation $\pi$, let $\pi(A)=\{\pi(a) \mid a \in A\}$. For any $A \in \Sigma$, we say that $A$ is symmetric if $A=\pi(A)$ for all permutations $\pi$ over $N$.

For all $A \in \Sigma$ and all $i \in N$, let $m_{i}(A)=\max \left\{a_{i} \mid\left(a_{1}, \cdots, a_{i}, \cdots, a_{n}\right) \in\right.$ $A\} . m(A) \equiv\left(m_{i}(A)\right)_{i \in N}$ is the ideal point of $\mathrm{A}$.

Definition 1: A bargaining solution $F$ over $\Sigma$ is the Nash solution if for all $A \in \Sigma, F(A)=\left\{a \in A \mid \prod_{i \in N} a_{i} \geq \prod_{i \in N} x_{i}\right.$ for all $\left.x \in A\right\}$.

Denote the Nash solution by $F^{N}$. Note that, for nonconvex bargaining problems, the Nash solution is typically multi-valued.

\section{Basic axioms and their implications}

In this section, we present several standard axioms and examine their implications for solutions to bargaining problems. We begin by introducing three axioms, Efficiency, Anonymity and Scale Invariance, which are standard in the literature on Nash bargaining problems.

Efficiency (E): For any $A \in \Sigma$ and any $a \in F(A)$, there is no $x \in A$ such that $x>a$.

Anonymity (A): For any $A \in \Sigma$, if $A$ is symmetric, then $[a \in F(A) \Rightarrow$ $\pi(a) \in F(A)$ for any permutation $\pi$ over $N]$.

Scale Invariance (SI): For all $A \in \Sigma$ and all $\alpha \in \mathbf{R}_{++}^{n}$, if $\alpha A=\left\{\left(\alpha_{i} a_{i}\right)_{i \in N} \mid\right.$ $a \in A\}$, then $F(\alpha A)=\left\{\left(\alpha_{i} a_{i}\right)_{i \in N} \mid a \in F(A)\right\}$. 
The axiom (A) is a weak equity condition reflecting the idea of equal treatment for equals. The axiom (SI) is typically justified in the context of convex bargaining problems by appealing to the expected utility theory and the possibility of randomization of actions. However, as Zhou (1997), $\mathrm{Xu}$ and Yoshihara (2006), and Peters andVermeulen (2012) have noted, the randomization is often unavailable due to the lack of correlating strategies among players even when players have cardinally measurable utility functions. Such cases correspond to nonconvex bargaining problems where (SI) can be interpreted as the axiom of "utility-unit invariance", and/or the axiom of "independence of utility intensities" as discussed in Yoshihara (2003) and $\mathrm{Xu}$ and Yoshihara (2006).

The next axioms represent minimal requirements on bargaining solutions to exhibit a consistent behavior. The requirements are in the spirit of the well-known Weak Axiom of Revealed Preference (WARP) used in the theory of revealed preference (Samuelson $(1938,1947)$ ), but is considerably weaker than WARP. The standard (WARP) such as defined in Sen (1971) requires that if an alternative $z$ is "revealed to be worse" than another alternative $x$ in the sense that there is a problem containing $x$ and $z$ from which $x$ is chosen but $z$ is not, then $z$ should not be chosen from any problem as a solution as long as $x$ is available. We propose two much weaker versions of (WARP) in this paper and are formally stated as follows:

Binary Weak Axiom of Revealed Preference 1 (BWARP1): For all $x, y, z \in \mathbf{R}_{+}^{n}$ with $y>z$, if $\{x, y\}=F(\operatorname{comp}\{x, y\})$, then $z \notin F(A)$ for any $A \in \Sigma$ with $x, z \in A$.

Binary Weak Axiom of Revealed Preference 2 (BWARP2): For all $x, z \in \mathbf{R}_{+}^{n}$, if $\{x\}=F(\operatorname{comp}\{x, z\})$, then $z \notin F(A)$ for any $A \in \Sigma$ with $x, z \in A$.

(BWARP1) requires that, if an alternative $z$ is "revealed to be worse" than another alternative $x$ through a "pairwise comparison" involving $x$ and $y$ where $y$ vectorially dominates $z$, then $z$ should not be chosen as a solution as long as $x$ is available. (BWARP2), on the other hand, requires that, if an alternative $z$ is "revealed to be worse" than another alternative $x$ via a "pairwise comparison" involving $x$ and $z$ directly, then $z$ should not be chosen as a solution as long as $x$ is available.

One attractive feature of (BWARP1) and (BWARP2) is their simplicity: it only involves the information about the solution to one type of simple bar- 
gaining problems. (BWARP1) and (BWARP2) represent a weak property of rational choice: it can be checked that, if a solution satisfies (E), (BWARP1) and (BWARP2), then there is an acyclic relation $P$ over $\mathbf{R}_{+}^{n}$ such that for any $A \in \Sigma,[x \in F(A) \Rightarrow \nexists y \in A$ s.t. $y P x]$.

We now explore the implications of the above axioms that are to be imposed on a solution to nonconvex bargaining problems. Our result in this section shows that, when a solution satisfies (A) and (SI), then it satisfies (E), (BWARP1) and (BWARP2) if and only if it selects from the Nash solution and coincides with the Nash solution on simple problems.

Proposition 1. Let a solution F satisfy (A) and (SI). Then, the following two statements are equivalent

(1.1) $F$ satisfies (E), (BWARP1) and (BWARP2),

(1.2) $F$ selects from $F^{N}$, and coincides with $F^{N}$ on simple problems.

Proof. We first show that (1.1) implies (1.2). For this purpose, suppose that $F$ satisfies (E), (BWARP1) and (BWARP2). We first establish the following two statements:

(1.3) $\forall x, y \in \mathbf{R}_{+}^{n}: \prod_{i \in N} x_{i}=\prod_{i \in N} y_{i}>0 \Rightarrow F(\operatorname{comp}\{x, y\})=\{x, y\}$,

(1.4) $\forall x, y \in \mathbf{R}_{+}^{n}: \prod_{i \in N} x_{i}>\prod_{i \in N} y_{i} \geq 0 \Rightarrow F(\operatorname{comp}\{x, y\})=\{x\}$.

Let $x, y \in \mathbf{R}_{+}^{n}$.

Consider $\prod_{i \in N} x_{i}=\prod_{i \in N} y_{i}>0$. Choose an appropriate $\alpha \in \mathbf{R}_{++}^{n}$ such that $\alpha x$ and $\alpha y$ are permutations of each other (such $\alpha$ always exists because $x$ and $y$ have the same value of their respective Nash product). Let $S \equiv$ $\operatorname{comp}\{\alpha x, \alpha y\}$. Then, let $T \equiv \cup_{\pi \in \Pi} \pi(S)$ where $\Pi$ is the set of all permutations of $N$. By construction, $T$ is symmetric, and $\{\pi(\alpha x), \pi(\alpha y) \mid \pi \in \Pi\} \subseteq$ $T$ is the set of all efficient outcomes in $T$. Thus, $F(T) \subseteq\{\pi(\alpha x), \pi(\alpha y) \mid \pi \in \Pi\}$, and let $\alpha x \in F(T)$. Then, by (A), $\{\pi(\alpha x) \mid \pi \in \Pi\} \subseteq F(T)$. Also, since $\alpha x$ and $\alpha y$ are permutations of each other, $\alpha y \in F(T)$ by (A). Then, again by (A), $\{\pi(\alpha y) \mid \pi \in \Pi\} \subseteq F(T)$. Thus, $F(T)=\{\pi(\alpha x), \pi(\alpha y) \mid \pi \in \Pi\}$, implying that $\alpha x, \alpha y \in F(T)$. Then, by (E) and (BWARP1), $\{\alpha x, \alpha y\}=$ $F(S)$. By (SI), $F(\operatorname{comp}\{x, y\})=\{x, y\}$. This shows that (1.3) holds.

Next, consider $\prod_{i \in N} x_{i}>\prod_{i \in N} y_{i} \geq 0$. Then, by choosing an appropriate $\varepsilon \in \mathbf{R}_{+}^{n}$ with $\epsilon>0$, we can have $\prod_{i \in N} x_{i}=\prod_{i \in N} z_{i}$ for $z \equiv y+\varepsilon$. From (1.3), $F(\operatorname{comp}\{x, z\})=\{x, z\}$. Noting that $z>y$ and by (BWARP2), 
$y \notin F(\operatorname{comp}\{x, y\})$. Thus, by $(\mathrm{E}), F(\operatorname{comp}\{x, y\})=\{x\}$. This proves that (1.4) holds.

Now, take any $A \in \Sigma$ and $x \in F(A)$. Suppose $x \notin F^{N}(A)$. Then, there exists $y \in F^{N}(A)$ such that $\prod_{i \in N} y_{i}>\prod_{i \in N} x_{i} \geq 0$. By (1.4), $F(\operatorname{comp}\{x, y\})=\{y\}$. Noting that $y \in A$, by (BWARP2), $x \notin F(A)$, which is a contradiction.

The above establishes that (1.1) implies (1.2).

To see that (1.2) implies (1.1), we first note that any selection from $F^{N}$ satisfies (E). Therefore, we need only to show that, if $F$ selects from $F^{N}$, and coincides with $F^{N}$ on simple problems, then $F$ satisfies (BWARP1) and (BWARP2).

Let $F$ select from $F^{N}$, and coincides with $F^{N}$ on simple problems.

$F$ satisfies (BWARP1): Let $x, y, z \in \mathbf{R}_{+}^{n}$ with $y>z$, and $\{x, y\}=$ $F(\operatorname{comp}\{x, y\})$. Since $F$ coincides with $F^{N}$ on simple problems, we have

$$
\{x, y\}=F(\operatorname{comp}\{x, y\})=F^{N}(\operatorname{comp}\{x, y\})
$$

Then, $\prod_{i \in N} x_{i}=\prod_{i \in N} y_{i}>\prod_{i \in N} z_{i}$. Consider any $A \in \Sigma$ with $x, z \in A$. Then, $z \notin F^{N}(A)$. Note that $F$ selects from $F^{N}$. We then have $z \notin F(A)$.

$F$ satisfies (BWARP2): Let $x, z \in \mathbf{R}_{+}^{n}$ and $\{x\}=F(\operatorname{comp}\{x, z\})$. Since $F$ coincides with $F^{N}$ on simple problems, we then have $\prod_{i \in N} x_{i}>\prod_{i \in N} z_{i}$. Consider any $A \in \Sigma$ with $x, z \in A$. Then $z \notin F^{N}(A)$. Then, $z \notin F(A)$ since $F$ selects from $F^{N}$.

Therefore, we have shown that (1.2) implies (1.1). $\diamond$

Our Proposition 1 can be regarded as a partial characterization of the Nash solution to nonconvex bargaining problems. It also shows that if we are interested in recommending an efficient and 'equitable' solution having some (weak) property of rational choice, we must choose from the set of options contained in the Nash solution.

\section{Proposing a refinement of the Nash solu- tion}

Given Proposition 1 in the last section, if we are interested in solutions having certain properties like (E), (A), (SI), (BWARP1) and (BWARP2), then we are confined to considering alternatives recommended by the Nash 
solution. Given the multi-valuedness of the Nash solution, the Nash solution is not necessarily attractive as it typically contains many undesirable outcomes in terms of fairness. Consider the following bargaining problem with $A=\operatorname{comp}\{(3,12),(6,6),(9,4)\}$. It can be easily checked that $F^{N}(A)=$ $\{(3,12),(6,6),(9,4)\}$. Note that, for both players, the outcome $(6,6)$ lies 'in-between' $(3,12)$ and $(9,4)$ since for player 1 , we have $3<6<9$ and for player 2 , we have $4<6<12$. In a sense, the outcome $(6,6)$ is 'more equitable' than the two 'extreme' outcomes $(3,12)$ and $(9,4)$. Based on this intuition, we shall introduce a refinement of the Nash solution to nonconvex problems. Our refinement of the Nash solution selects, for each bargaining problem $A$, all the points in $F^{N}(A)$ that also lie on the highest indifference surface given by $\min \left(a_{1} / m_{1}(A), \cdots, a_{n} / m_{n}(A)\right)$ attainable in $A .^{2}$ Formally, we have:

Definition 2: A bargaining solution over $\Sigma$ is the equitable Nash solution, to be denoted by $F^{E N}$, if for all $A \in \Sigma, F^{E N}(A)=\left\{a \in F^{N}(A)\right.$ $\min _{i \in N}\left\{\frac{a_{i}}{m_{i}(A)}\right\} \geq \min _{i \in N}\left\{\frac{x_{i}}{m_{i}(A)}\right\}$ for all $\left.x \in F^{N}(A)\right\}$.

Therefore, for any given problem $A$, to obtain $F^{E N}(A)$, we first find out the Nash solution, $F^{N}(A)$, to the bargaining problem $A$, and then, from the Nash solution set $F^{N}(A)$, we select all the points lying on the highest indifference surface given by $\min \left(a_{1} / m_{1}(A), \cdots, a_{n} / m_{n}(A)\right)$.

To see that $F^{E N}$ is a (proper) refinement of the Nash solution, we consider our earlier bargaining problem $A=\operatorname{comp}\{(3,12),(6,6),(9,4)\}$. It may be checked that $F^{N}(A)=\{(3,12),(6,6),(9,4)\}$, whereas $F^{E N}(A)=\{(6,6)\}$.

Note that, for convex bargaining problems, $F^{E N}$ coincides with the Nash solution $F^{N}$. Therefore, $F^{E N}$ is an extension of the standard Nash solution defined for convex problems.

It is worth mentioning that $F^{E N}$ violates a continuity property defined below:

\footnotetext{
${ }^{2}$ The KS solution can be defined as usual: for all $A \in \Sigma, F^{K S}(A)=\{x \in A \mid$ $x_{1} / m_{1}(A)=\cdots=x_{n} / m_{n}(A)$, and there exists no $y \in A$ such that $\left.y \gg x\right\}$. It may be noted that the solution given by $F(A)=\left\{a \in A \mid \min \left(a_{1} / m_{1}(A), \cdots, a_{n} / m_{n}(A)\right) \geq\right.$ $\left.\min \left(x_{1} / m_{1}(A), \cdots, x_{n} / m_{n}(A)\right), \forall x \in A\right\}$ for all $A \in \Sigma$ is a natural extension of the KS solution to nonconvex problems, as proposed by Nagahisa and Tanaka (2002). The reason that we use this variant of the KS solution (instead of the KS solution itself) is that $F^{N}(A) \cap F^{K S}(A)=\varnothing$ for some $A \in \Sigma$.
} 
Continuity (CON): For any sequence $\left\{A^{k}\right\} \subseteq \Sigma$ which converges to $A^{0}$ in the sense of the Hausdorf metric, and for any sequence $\left\{x^{k}\right\}$ such that $x^{k} \in F\left(A^{k}\right)$ for each $k$ and $x^{k} \rightarrow x^{0}, x^{0} \in F\left(A^{0}\right)$ holds.

To see that $F^{E N}$ violates $(\mathrm{CON}),{ }^{3}$ consider again our earlier bargaining problem $A=\operatorname{comp}\{(3,12),(6,6),(9,4)\}$. Note that $F^{E N}=\{(6,6)\}$. Let $\epsilon>0$ and define

$$
A(\epsilon)=\operatorname{comp}\{(3,12),(6-\epsilon, 6-\epsilon),(9,4)\}
$$

Then, $F^{E N}(A(\epsilon))=F^{N}(A(\epsilon))=\{(3,12),(9,4)\}$, which does not converge to $\{(6,6)\}$.

Note that there exists no single-valued solution to nonconvex problems which satisfies (CON) and refines the Nash solution at the same time, as was shown by Peters and Vermeulen (2012) and Xu and Yoshihara (2013). Thus, every single-valued refinement of (asymmetric) Nash solution proposed by Peters and Vermeulen (2012) violates (CON) and (AN), whereas any other proposals of extensions of the Nash solution of convex problems to nonconvex problems such as Zhou (1997) and Conley and Wilkie (1998) violate either $(\mathrm{AN})$ or $(\mathrm{E})$ although their proposals are single-valued and satisfy $(\mathrm{CON})$.

\section{Characterization of $F^{E N}$}

In this section, we study the behavior of the solution $F^{E N}$. We begin by introducing a notation. For all $x \in \mathbf{R}_{+}^{n}$, let $\bar{x}=\max \left\{x_{i} \in \mathbf{R}_{+} \mid i=1, \cdots, n\right\}$ and $\underline{x}=\min \left\{x_{i} \in \mathbf{R}_{+} \mid i=1, \cdots, n\right\}$. Next, we note that $F^{E N}$ satisfies the following two axioms.

Equity Principle (EP): For all $A \in \Sigma$ such that $A$ is not a simple problem, is symmetric, and for all $x \in A$, if there exists $y \in A$ such that $\{x, y\}=$ $F(\operatorname{comp}\{x, y\})$ and $(\underline{x}, \cdots, \underline{x})<y<(\bar{x}, \cdots, \bar{x})$, then $x \notin F(A)$.

Weak Contraction Independence (WCI): For any $A, B \in \Sigma$, if $m(A)=$ $m(B), A \subseteq B$ and $A \cap F(B) \neq \varnothing$, then $F(A)=F(B) \cap A$.

\footnotetext{
${ }^{3}$ We are grateful to an Associate Editor for pointing this out and for suggesting the example below.
} 
(EP) reflects an equity concern in making a solution recommendation for bargaining problems. It says that, for any symmetric problem $A$ and for any two points, $x$ and $y$, in $A$, if, in the simple bargaining problem formed by the comprehensive hull of $x$ and $y$, they both are chosen as the solution to the problem $\operatorname{comp}\{x, y\}$, but the maximum (resp. minimum) utility specified in $x$ is greater (resp. less) than the maximum (resp. minimum) utility specified in $y$ (so that the utility distribution under $y$ is more evenly distributed than under $x$ ), then $x$ should not be chosen from $A$. It may be noted that this equity idea is originated from the Hammond equity (Hammond (1976)) which is embedded in the requirements that $(\underline{x}, \cdots, \underline{x})<y<(\bar{x}, \cdots, \bar{x})$ and that $A$ is a symmetric problem. Note that a typical underlying environment from which a symmetric problem arises is that every player has the same preference relation over social alternatives and they have the same physical option set. In such a case, an idea of inequality aversion in outcomes such as the Hammond equity seems reasonable from a viewpoint of equal treatment of equals. Moreover, (EP) also incorporates our intuition that a procedure of sequential binary choices is not sufficient to make an equitable recommendation, in that it is typical that a solution keeps silent for comparing $x$ and $y$ in the simple problem $\operatorname{comp}\{x, y\}$. In such a case, the solution should refer to a global information of the problem $A$ to apply an equity consideration.

(WCI) is a familiar one used for characterizing the Kalai-Smordinsky (KS) solution for nonconvex bargaining problems (see, for example, $\mathrm{Xu}$ and Yoshihara (2006)). As argued in Xu and Yoshihara (2008), the axiom (WCI) can be interpreted as reflecting an idea of solidarity in making solution recommendations. Indeed, the solidarity idea embedded in (WCI) is that, whenever a problem $B$ shrinks to another problem $A$ while the ideal positions for players are unchanged, the change in each player's compromising from her ideal position should be adjusted to the same direction as any other player's. Hence, as long as a subset of $F(B)$ is still available in $A$, this subset $F(B) \cap A$ should continue to be the solution to $A$ due to the spirit of solidarity. This requirement is particularly relevant when a solution is efficient, since any movement with the same direction away from any alternative in $F(B) \cap A$ will make every player worse off.

With the help of axioms (EP) and (WCI), we obtain the following result, which is a characterization of $F^{E N}$.

Theorem 1. A solution F satisfies (E), (A), (SI), (BWARP1), (BWARP2), (WCI), and (EP) if and only if $F=F^{E N}$. 
Proof. It can be checked that $F^{E N}$ satisfies (E), (A), (SI), (BWARP1), (BWARP2), (WCI), and (EP). We now show that a solution $F$ satisfying (E), (A), (SI), (BWARP1), (BWARP2), (WCI), and (EP) must be $F^{E N}$. Let $F$ satisfy (E), (A), (SI), (BWARP1), (BWARP2), (WCI), and (EP).

Take any $A \in \Sigma$ and $x^{*} \in F^{E N}(A)$. By Proposition 1, $F(A) \subseteq F^{N}(A)$. Take any $y \in F^{N}(A) \backslash F^{E N}(A)$, and suppose $y \in F(A)$. Consider $\alpha \in$ $\mathbf{R}_{++}^{n}$ such that $m_{i}\left(A^{\prime}\right)=m_{j}\left(A^{\prime}\right)$ for any $i, j \in N$, where $A^{\prime} \equiv \alpha A$. Let $x^{* \prime} \equiv \alpha x^{*}$. Then, by (SI), $y^{\prime} \in F\left(A^{\prime}\right)$, where $y^{\prime} \equiv \alpha y$. Consider $S \equiv$ $\operatorname{comp}\left\{x^{* \prime}, y^{\prime},\left(m_{1}\left(A^{\prime}\right), \mathbf{0}_{-1}\right), \ldots,\left(m_{n}\left(A^{\prime}\right), \mathbf{0}_{-n}\right)\right\} \subseteq A^{\prime}$. Then, by (WCI), $y^{\prime} \in F(S)$. Also consider $S^{\prime} \equiv \operatorname{comp}\left\{x^{* \prime}, y^{\prime}\right\}$. Then, by Proposition 1., $\left\{x^{* \prime}, y^{\prime}\right\}=F\left(S^{\prime}\right)$. Next, consider $T \equiv \cup_{\pi \in \Pi} \pi(S)$. By (A), (WCI), and (E), $\left\{\pi\left(y^{\prime}\right)\right\}_{\pi \in \Pi} \subseteq F(T)$, where $\Pi$ is the set of all permutations of $N$. Note that $S^{\prime} \cup T=T$ is symmetric and $\left\{x^{* \prime}, y^{\prime}\right\}=F\left(\operatorname{comp}\left\{x^{* \prime}, y^{\prime}\right\}\right)$. Let $\bar{y}^{\prime} \equiv\left(\bar{y}^{\prime}, \cdots, \bar{y}^{\prime}\right)$ and $\underline{y}^{\prime} \equiv\left(\underline{y}^{\prime}, \cdots, \underline{y}^{\prime}\right)$. Then, since $x^{* \prime} \in F^{E N}\left(A^{\prime}\right)$ and $y^{\prime} \in F^{N}\left(A^{\prime}\right) \backslash F^{E N}\left(A^{\prime}\right)$, we have $\bar{y}^{\prime}>x^{* \prime}>y^{\prime}$. Thus, by (EP) and (A), $\left\{\pi\left(y^{\prime}\right)\right\}_{\pi \in \Pi}$ cannot be a subset of $F(T)$, which is a contradiction. Thus, for any $y \in F^{N}(A) \backslash F^{E N}(A), y \notin F(A)$. Combining with Proposition 2, we conclude that $F(A) \subseteq F^{E N}(A)$. Finally, we can see that $F(A)=F^{E N}(A)$ by $(\mathrm{SI})$ and $(\mathrm{A}) . \diamond$

From Theorem 1, it may be noted that many axioms used for characterizing $F^{E N}$ are also satisfied by the KS solution to nonconvex problems. It can be checked that the KS solution to nonconvex problems can be characterized by the properties of weak efficiency and single-valuedness together with (A), (SI), and (WCI) (see, for example, Xu and Yoshihara (2006) and Lombardi and Yoshihara (2010)). Moreover, the KS solution satisfies (CON) and (EP). Note that the KS solution violates (E), (BWARP1) and (BWARP2), since the $\mathrm{KS}$ solution is not rationalizable by any acyclic binary relation. In contrast, though $F^{E N}$ is rationalizable by an acyclic binary relation, it is not single-valued. These observations suggest that, in the presence of the properties like (E), (A), (SI), (BWARP1) and (BWARP2), rationalization of a solution by an acyclic relation and the single-valuedness of a solution are not compatible for nonconvex bargaining problems.

To conclude this section, we discuss the independence of the axioms figured in Theorem 1 by focusing on (BWARP1), (BWARP2), (EP) and (WCI). Note that the following solution $F^{p N}$ : for all $A \in \Sigma, F^{p N}(A)=$ $\left\{a \in F^{N}(A) \mid \min _{i \in N}\left\{\frac{a_{i}}{m_{i}\left(\operatorname{comp} F^{N}(A)\right)}\right\} \geq \min _{i \in N}\left\{\frac{x_{i}}{m_{i}\left(\operatorname{comp} F^{N}(A)\right)}\right\}\right.$ for all $x \in$ 
$\left.F^{N}(A)\right\}$ satisfies $(\mathrm{E}),(\mathrm{A}),(\mathrm{SI}),(\mathrm{BWARP} 1),(\mathrm{BWARP} 2)$ and $(\mathrm{EP})$, but violates (WCI). Note that $F^{N}$ satisfies (E), (A), (SI), (BWARP1), (BWARP2) and (WCI), but violates (EP). Finally, consider the following solution $F^{P}$ : for all $A \in \Sigma$, let $P(A)$ be the Pareto set of $A$, and let $F^{P}(A)=\{a \in P(A) \mid$ $\min _{i \in N}\left\{\frac{a_{i}}{m_{i}(A)}\right\} \geq \min _{i \in N}\left\{\frac{x_{i}}{m_{i}(A)}\right\}$ for all $\left.x \in P(A)\right\}$. It can be checked that $F^{P}$ satisfies (E), (A), (SI), (EP) and (WCI) but violates (BWARP1) and (BWARP2).

\section{Conclusion}

In this paper, we have introduced a new solution as an equitable refinement of the Nash solution to nonconvex bargaining problems and studied it axiomatically. The following table summarizes the properties of the Nash, KS, $F^{E N}$ solutions discussed in the paper where o indicates the specified solution satisfies the corresponding axiom while $\times$ indicates otherwise:

$\begin{array}{cccc} & \underline{N} & \underline{K S} & \underline{F^{E N}} \\ (E) & \circ & \times & \circ \\ (A) & \circ & \circ & \circ \\ (S I) & \circ & \circ & \circ \\ (B W A R P 1) & \circ & \times & \circ \\ (B W A R P 2) & \circ & \times & \circ \\ (E P) & \times & \circ & \circ \\ (W C I) & \circ & \circ & \circ\end{array}$

Note that the equitable Nash solution proposed in this paper can be implemented by the two-stage bargaining procedure: to arrive at the final recommendation for nonconvex bargaining problems, it first employs the Nash solution concept and - among the options recommended by the Nash solution - then it adopts a procedure based on the idea of the KS solution with reference to an ideal point. Such a two-stage procedure resembles the idea of sequential rationalizability discussed in the context of abstract choice problems by Manzini and Mariotti (2006, 2007) and by Tadenuma (2002). However, there is a significant difference between the two-stage procedure of the equitable Nash solution and the notion of sequential rationalizability. The difference is that $F^{E N}$ (to abuse the notion, we interpret $F^{E N}$ as a choice 
function here) cannot be a sequentially rationalizable choice function. To see this, for a given binary relation $R \subseteq \mathbf{R}_{+}^{n} \times \mathbf{R}_{+}^{n}$ with $P(R)$ as its corresponding strict part, let $\max (A ; R) \equiv\{x \in A \mid \forall y \in A:(y, x) \notin P(R)\}$. Then, a choice function $C$ is sequentially rationalizable if there exist two binary relations $R_{1}, R_{2}$ over $\mathbf{R}_{+}^{n}$ such that, for any $A \in \Sigma, C(A)=\max \left(\max \left(A ; R_{1}\right) ; R_{2}\right)$. Now, let $R^{N} \subseteq \mathbf{R}_{+}^{n} \times \mathbf{R}_{+}^{n}$ be a binary relation such that for any $x, y \in \mathbf{R}_{+}^{n}$, $(x, y) \in R^{N}$ if and only if $\prod_{i \in N} x_{i} \geq \prod_{i \in N} y_{i}$. If $F^{E N}$ is a sequentially rationalizable choice function, then there must be another binary relation $R^{E} \subseteq \mathbf{R}_{+}^{n} \times \mathbf{R}_{+}^{n}$ such that for any $A \in \Sigma, F^{E N}(A)=\max \left(\max \left(A ; R^{N}\right) ; R^{E}\right)$. Note, however, $F^{E N}(A)=\max \left(\max \left(A ; R^{N}\right) ; R_{A}^{E}\right)$ for any $A \in \Sigma$, where $R_{A}^{E} \subseteq \mathbf{R}_{+}^{n} \times \mathbf{R}_{+}^{n}$ is such that for any $x, y \in \mathbf{R}_{+}^{n},(x, y) \in R_{A}^{E}$ if and only if $\min _{i \in N}\left\{\frac{x_{i}}{m_{i}(A)}\right\} \geq \min _{i \in N}\left\{\frac{y_{i}}{m_{i}(A)}\right\}$. This suggests that $R_{A}^{E}$ may vary according to the feasible set $A$, which implies that $F^{E N}$ may not be sequentially rationalizable.

On the other hand, the endogenous nature of the underlying second criterion $R_{A}^{E}$ is perhaps a desired feature of $F^{E N}$. This is because $R_{A}^{E}$ represents an ethical principle in terms of procedural fairness stipulating what social outcomes should be selected from which feasible option set. In $F^{E N}$, the necessary information about feasible option sets for identifying the ranking $R_{A}^{E}$ is of $m(A)$.

We offer a final remark to conclude the paper. It may be of interest to note that our framework can be readily adapted to studying collective choice problems where each bargaining problem can be interpreted as a feasible set available to a fixed number of individuals in the society and a solution can be interpreted as a collective choice function. With this reinterpretation, the axioms employed in the paper can be reformulated and re-interpreted accordingly. This seems to open a new possibility of investigating and studying collective choice problems. It would be interesting to explore this possibility in various contexts.

\section{References}

1. Conley, J. and S. Wilkie (1991): "The bargaining problem without convexity: extending the egalitarian and Kalai-Smorodinsky solutions," Economics Letters 36, 365-369.

2. Conley, J. and S. Wilkie (1996): "An extension of the Nash bargaining 
solution to nonconvex problems," Games and Economic Behavior 13, 26-38.

3. Hammond, P. (1976): "Equity, Arrow's conditions, and Rawls' difference principle," Econometrica 44, 793-804.

4. Herrero, M. J. (1989): "The Nash program: non-convex bargaining problems," Journal of Economic Theory 49, 266-277.

5. Kalai, E. and M. Smorodinsky (1975): "Other solutions to Nash's bargaining problem," Econometrica 43, 513-518.

6. Lombardi, M. and N. Yoshihara (2010): "Alternative characterizations of the proportional solution for nonconvex bargaining problems with claims," Economics Letters 108, 229-232.

7. Manzini, P. and M. Mariotti (2006): "Two-stage bargaining solutions," Working Paper No. 572, University of London at Queen Mary.

8. Manzini, P. and M. Mariotti (2007): "Sequentially rationalizable choice," American Economic Review 97, 1824-1839.

9. Mariotti, M. (1998): "Nash bargaining theory when the number of alternatives can be finite," Social Choice and Welfare 15, 413-421.

10. Mariotti, M. (1999): "Fair bargains: distributive justice and Nash bargaining theory," Review of Economic Studies 66, 733-741.

11. Mariotti, M. (2000): "Maximal symmetry and the Nash solution," Social Choice and Welfare 17, 45-53

12. Nagahisa, R. and M. Tanaka (2002): "An axiomatization of the KalaiSmorodinsky solution when the feasible sets can be finite," Social Choice and Welfare 19, 751-761.

13. Nash, J. F. (1950): "The bargaining problem," Econometrica 18, 155162.

14. Peters, H.J.M. and D. Vermeulen (2012): "WPO, COV and IIA bargaining solutions for non-convex bargaining problems," International Journal of Game Theory 41, 851-884. 
15. Samuelson, P.A. (1938): "A note on the pure theory of consumer's behavior," Economica, 61-71.

16. Samuelson, P.A. (1947). Foundations of Economic Analysis (Cambridge, MA: Harvard University Press).

17. Sen, A.K. (1971): Choice functions and revealed preference, Review of Economic Studies 38, 307-317.

18. Tadenuma, K. (2002): "Efficiency first or equity first? Two principles and rationality of social choice," Journal of Economic Theory 104, 462472.

19. $\mathrm{Xu}, \mathrm{Y}$. and N. Yoshihara (2006): "Alternative characterizations of three bargaining solutions for nonconvex problems," Games and Economic Behavior 57, 86-92.

20. Xu, Y. and N. Yoshihara (2008): "The Behavior of Solutions to Bargaining Problems on the Basis of Solidarity," Japanese Economic Review 57(1), 86-92.

21. Xu, Y. and N. Yoshihara (2013): "Rationality and solutions to nonconvex bargaining problems: rationalizability and Nash solutions," Mathematical Social Sciences 66, 66-70.

22. Yoshihara, N. (2003): "Characterizations of bargaining solutions in production economies with unequal skills," Journal of Economic Theory 108, 256-285.

23. Zhou, L. (1997): "The Nash bargaining theory with non-convex problems," Econometrica 65, 681-686. 